\title{
UNIFORMIZATION OF CERTAIN SHIMURA CURVES
}

\author{
PILAR BAYER \\ Facultat de Matemàtiques, Universitat de Barcelona \\ Gran Via de les Corts Catalanes 585, E-08007 Barcelona, Spain \\ E-mail:bayer@mat.ub.es
}

\begin{abstract}
We present an approach to the uniformization of certain Shimura curves by means of automorphic functions, obtained by integration of non-linear differential equations. The method takes as its starting point a differential construction of the modular $j$-function, first worked out by R. Dedekind in 1877, and makes use of a differential operator of the third order, introduced by H. A. Schwarz in 1873 .
\end{abstract}

Introduction. Shimura curves afford a notable generalization of classical modular curves. Whereas modular curves appear as moduli spaces of elliptic curves, Shimura curves appear as moduli spaces of principally polarized abelian surfaces endowed with quaternionic multiplication. From an algorithmic point of view, the fact that elliptic curves are much better understood than abelian surfaces means that there is a fundamental difference between the treatment of the two topics.

The uniformization of Shimura curves and the computation of their automorphic forms have been long-standing problems. In general, algorithms used in the modular case do not transfer directly to the quaternionic case. The main difficulties are due to the fact that while fuchsian groups defining modular curves contain translations, fuchsian groups defining Shimura curves lack them. In consequence, automorphic forms and automorphic functions will possess Fourier developments only in the modular case.

An oft-quoted example of automorphic function is the modular $j$-function. We provide an outline of a differential construction of it, which goes back to R. Dedekind [3], based on the integration of a fuchsian differential equation of the second order and Jacobi's theory of elliptic integrals.

2000 Mathematics Subject Classification: Primary 11G18; Secondary 14 G35.

Key words and phrases: Shimura curve, fundamental domain, fuchsian differential equation, schwarzian derivative.

Partially supported by MCYT BFM2000-0627.

The paper is in final form and no version of it will be published elsewhere. 
The importance of Dedekind's construction of the $j$-function lies in the fact that, in principle, it can be adapted to uniformize Shimura curves. However, it should be stressed that to carry out this programme it is necessary to have previous knowledge of a fundamental domain of the curve and additional information as well, since the uniformizing differential equation cannot be determined directly from a fundamental domain.

M. Alsina constructed fundamental domains for some Shimura curves in [1]. The theoretical framework of this construction consists of an interplay between arithmetic properties of Eichler orders and (binary, ternary and quaternary) quadratic forms of integral (or algebraic integral) coefficients.

The results presented here are from an ongoing project carried out jointly with A. Travesa and J. Guàrdia. Fuller discussions will appear in later publications.

It is worth noting that Shimura curves have received a great deal of attention in recent years, mainly due to their role in A. Wiles' proof of Fermat's Last Theorem.

1. Fuchsian groups of the first kind. We first recall some basic definitions concerning the hyperbolic 2 -space. We consider the Riemann sphere $\mathbb{P}^{1}=\mathbb{C} \cup\{\infty\}$. The special linear group $\mathbf{S L}(2, \mathbb{C})$, of complex $2 \times 2$-matrices with determinant equal to one, acts naturally on $\mathbb{P}^{1}$ as a group of homographic transformations. An element $\alpha=\left[\begin{array}{ll}a & b \\ c & d\end{array}\right]$ of $\mathbf{S L}(2, \mathbb{C})$ acts as

$$
\alpha(z)=\frac{a z+b}{c z+d}, \quad \text { for } z \in \mathbb{P}^{1} .
$$

This action factorizes through the projective linear group $\mathbf{P S L}(2, \mathbb{C})=\mathbf{S L}(2, \mathbb{C}) /\langle-\mathrm{I}\rangle$. All the directly conformal homeomorphisms of $\mathbb{P}^{1}$ are obtained in this way.

The complex upper-half plane, $\mathcal{H}=\{z \in \mathbb{C}: \operatorname{Im}(z)>0\}$, is a reference model for the hyperbolic 2 -space, once it is endowed with the hyperbolic distance

$$
d\left(z_{1}, z_{2}\right)=\left|\operatorname{arccosh}\left(1+\frac{\left|z_{1}-z_{2}\right|^{2}}{2 z_{1} z_{2}}\right)\right|,
$$

and the $\mathbf{S L}(2, \mathbb{R})$-invariant 2 -form $d\left(y^{-1} d x\right)=y^{-2} d x \wedge d y$. The groups $\mathbf{S L}(2, \mathbb{R})$ and $\mathbf{P S L}(2, \mathbb{R})=\mathbf{S L}(2, \mathbb{R}) /\langle-\mathrm{I}\rangle$ act on $\mathcal{H}$, as already stated, and yield all the orientationpreserving isometries of $\mathcal{H}$.

On the basis of the number and nature of their fixed points, homographic transformations $\alpha \in \mathbf{S L}(2, \mathbb{R}) \backslash\langle-\mathrm{I}\rangle$ are classified in three types: hyperbolic, elliptic and parabolic. Hyperbolic transformations $(|\operatorname{tr}(\alpha)|>2$ ) have two fixed points, both lying on $\mathbb{R} \cup\{\infty\}$. Elliptic transformations $(|\operatorname{tr}(\alpha)|<2)$ have two fixed points, which are complex conjugate; one of them lies in $\mathcal{H}$. Parabolic transformations $(|\operatorname{tr}(\alpha)|=2)$ have one fixed point exactly, located on $\mathbb{R} \cup\{\infty\}$. The corresponding Jordan forms of these transformations are of the shape:

$$
\left[\begin{array}{cc}
\lambda_{1} & \\
& \lambda_{2}
\end{array}\right], \lambda_{1} \neq \lambda_{2} ; \quad\left[\begin{array}{cc}
e^{i \theta} & \\
& e^{-i \theta}
\end{array}\right], \theta \in \mathbb{R} ; \quad\left[\begin{array}{cc} 
\pm 1 & 1 \\
0 & \pm 1
\end{array}\right] .
$$

Let us now fix a discrete subgroup $\Gamma \subseteq \mathbf{S L}(2, \mathbb{R})$. A point $z \in \mathcal{H}$ is called elliptic if it is the fixed point of an elliptic transformation. A point $z \in \mathbb{R} \cup\{\infty\}$ is called a cusp if it is the fixed point of a parabolic transformation. We denote by $P_{\Gamma}$ the set of cusps of $\Gamma$. $P_{\Gamma}$ might very well be the empty set. 
The group $\Gamma$ acts on the set of its cusps. Thus, it also acts on

$$
\mathcal{H}^{*}:=\mathcal{H} \cup P_{\Gamma} .
$$

Endowed with a suitable topology and complex structure, the quotient space $\Gamma \backslash \mathcal{H}^{*}$ becomes a Riemann surface and the projection mapping $\pi: \mathcal{H}^{*} \rightarrow \Gamma \backslash \mathcal{H}^{*}$ is analytic.

A group $\Gamma$ is defined as fuchsian of the first kind when the Riemann surface $\Gamma \backslash \mathcal{H}^{*}$ is compact.

By a theorem of Siegel, fuchsian groups of the first kind are those for which the hyperbolic volume of $\Gamma \backslash \mathcal{H}^{*}$ is finite. We shall assume henceforth that this is always the case.

Attached to the group $\Gamma$, there is a projective curve, $X(\Gamma)$, and a meromorphic mapping,

$$
j_{\Gamma}: \mathcal{H}^{*} \rightarrow X(\Gamma)(\mathbb{C}),
$$

which factorizes in a bi-rational isomorphism of $\Gamma \backslash \mathcal{H}^{*}$ into $X(\Gamma)(\mathbb{C})$.

For a point $w=\pi(z) \in \Gamma \backslash \mathcal{H}^{*}$, let $\bar{\Gamma}_{w} \subset \mathbf{P S L}(2, \mathbb{R})$ denote its isotropy group under the $\Gamma$-action and $e_{w}=\sharp \bar{\Gamma}_{w}$ its order. We have that $e_{w}=\infty$ if $z$ is a cusp; $1<e_{w}<\infty$ if $z$ is an elliptic point; and $e_{w}=1$, otherwise. The genus $g$ of $X(\Gamma)$ can be calculated through the formula

$$
\frac{1}{2 \pi} \operatorname{vol}\left(\Gamma \backslash \mathcal{H}^{*}\right)=2 g-2+\sum_{w \in X(\Gamma)}\left(1-\frac{1}{e_{w}}\right) .
$$

Every subgroup $\Gamma^{\prime} \subseteq \Gamma$ of finite index gives rise to a finite morphism $\varphi: X\left(\Gamma^{\prime}\right) \rightarrow$ $X(\Gamma)$, of degree $n=\left[\overline{\bar{\Gamma}}: \bar{\Gamma}^{\prime}\right]$. The genus $g^{\prime}$ of $X\left(\Gamma^{\prime}\right)$ is obtained from the well-known Hurwitz formula:

$$
2 g^{\prime}-2=n(2 g-2)+\sum_{w \in X\left(\Gamma^{\prime}\right)}\left(e_{w, \varphi}-1\right), \text { where } e_{w, \varphi}:=\left[\bar{\Gamma}_{\varphi(w)}: \bar{\Gamma}_{w}^{\prime}\right] .
$$

Definition 1.1. By a fundamental domain for the $\Gamma$-action on $\mathcal{H}$ we mean a subset $\mathcal{D} \subseteq \mathcal{H} \cup \mathbb{R} \cup\{\infty\}$ such that

i) $\mathcal{D}$ is closed and connected,

ii) $\mathcal{H}=\bigcup_{\gamma \in \Gamma} \gamma(\mathcal{D})$,

iii) $\mathcal{D}=\bar{U}, U$ open set, $U=\operatorname{int}(\mathcal{D})$,

iv) $\gamma(U) \cap U=\emptyset$, for any $\gamma \in \Gamma, \gamma \neq \pm \mathrm{I}$.

If the shape of $\mathcal{D}$ is that of a hyperbolic polygon, then we call $\mathcal{D}$ a fundamental polygon for $\Gamma$.

Every fuchsian group $\Gamma$ possesses a fundamental polygon. In general, a great deal of information for $\Gamma \backslash \mathcal{H}^{*}$, or $X(\Gamma)$, may be deduced from a careful observation of a fundamental polygon.

2. Automorphic functions and automorphic forms. Put $\mathbf{G L}^{+}(2, \mathbb{R})=\{\alpha \in$ $\mathbf{G L}(2, \mathbb{R}) \mid \operatorname{det}(\alpha)>0\}$. Attached to an element $\alpha=\left[\begin{array}{ll}a & b \\ c & d\end{array}\right]$ of $\mathbf{G L}^{+}(2, \mathbb{R})$, an automorphic factor

$$
j(\alpha, z)=c z+d
$$

is defined; this is a holomorphic function on $\mathcal{H}$ without zeros. 
Let $k$ be an integer. For any function $f: \mathcal{H} \rightarrow \mathbb{P}^{1}$, we may consider the action

$$
\left(\left.f\right|_{k} \alpha\right)(z)=\operatorname{det}(\alpha)^{k / 2} j(\alpha, z)^{-k} f(\alpha z), \quad z \in \mathcal{H} .
$$

Fix a fuchsian group $\Gamma$. A meromorphic function $f(z)$ on $\mathcal{H}$ is called a $\Gamma$-automorphic form of weight $k$ if it is meromorphic at every cusp of $\Gamma$ and satisfies the functional equation

$$
\left.f\right|_{k} \gamma=f, \quad \text { for all } \gamma \in \Gamma .
$$

We denote by $\mathcal{A}_{k}(\Gamma)$ the $\mathbb{C}$-vector space of all $\Gamma$-automorphic forms of weight $k$.

Automorphic forms of weight zero are called automorphic functions. The field $\mathcal{A}_{0}(\Gamma)$ is the field of meromorphic functions of the Riemann surface $\Gamma \backslash \mathcal{H}^{*}$. It is therefore isomorphic to the field of rational functions on the corresponding projective curve:

$$
\mathcal{A}_{0}(\Gamma) \simeq \mathbb{C}(X(\Gamma)) .
$$

More generally, there is a one to one correspondence between automorphic forms of even weight $2 m$ on $\Gamma \backslash \mathcal{H}^{*}$ and meromorphic differential forms of degree $m$ on $X(\Gamma)$ :

$$
\mathcal{A}_{2 m}(\Gamma) \simeq \Omega^{m}(X(\Gamma)), \quad f \mapsto \omega_{f} .
$$

Here $\omega_{f}$ stands for the differential form which satisfies the equality $f(z)(d z)^{m}=\omega_{f} \circ \pi$.

When $\Gamma$ is contained in the modular group $\mathbf{S L}(2, \mathbb{Z})$, automorphic forms (respectively, functions) are simply designated as modular forms (respectively, modular functions).

The first example of a modular function for $\mathbf{S L}(2, \mathbb{Z})$ is provided by the $j$-function:

$$
j(z)=1728 \frac{g_{2}(z)^{3}}{\Delta(z)}, z \in \mathcal{H} .
$$

Here

$$
g_{2}(z)=60 \sum_{\substack{m, n \\(m, n) \neq(0,0)}} \frac{1}{(m+n z)^{4}}, \quad g_{3}(z)=140 \sum_{\substack{m, n \\(m, n) \neq(0,0)}} \frac{1}{(m+n z)^{6}}
$$

denote the Eisenstein series, which are modular forms of weights 4 and 6 , respectively, and

$$
\Delta(z)=g_{2}(z)^{3}-27 g_{3}(z)^{2}
$$

denotes the discriminant modular form, which is of weight 12 .

For a point $\tau \in \mathcal{H}$, the value $j(\tau)$ is an invariant of the elliptic curve

$$
E_{\tau}: Y^{2}=4 X^{3}-g_{2}(\tau) X-g_{3}(\tau) .
$$

The set $E_{\tau}(\mathbb{C})$ of all complex points of this curve is in one to one correspondence with those of the torus $\mathbb{C} /[1, \tau]$. The uniformization is carried over the Weierstraß functions:

$$
\mathbb{C} /[1, \tau] \simeq E_{\tau}(\mathbb{C}) \subset \mathbb{P}^{2}, \quad z \mapsto\left(\wp_{\tau}(z), \wp_{\tau}^{\prime}(z), 1\right) .
$$

3. A differential approach to automorphic functions. Although the topic seems to be practically forgotten today, the connection between automorphic functions and ordinary differential equations was much investigated in the past. Among the pioneer studies we find contributions of R. Dedekind and H. Poincaré. Both mathematicians were profoundly influenced in their research by the work of L. Fuchs [6] on ordinary differential equations. 
In 1877, Dedekind [3] constructed a special function, which he called Valenz, by integration of a second order differential equation of fuchsian type. Dedekind's valence function equals the function $j$, up to multiplication by a scalar.

The work of Poincaré on automorphic functions began in 1881 and was the focus of numerous publications collected in the second volume of his Euvres. To be exact, Poincaré did not denominate these functions automorphic but fuchsian, in Fuchs' honor. The name automorphic is due to F. Klein and is the term that has prevailed over the years.

The space $\mathcal{A}_{k}(\Gamma)$ of automorphic forms of weight $k$ is not closed under the usual derivation. Specifically, the derivative $D(f, z)$ of an automorphic function $f(z)$ is not an automorphic function but an automorphic form of weight two. The exact behaviour of an automorphic form of weight $k$ under derivation is given below.

Proposition 3.1. If $f(z)$ is an automorphic form of weight $k$, then

$$
k f(z) D^{2}(f, z)-(k+1) D(f, z)^{2}
$$

is an automorphic form of weight $2 k+4$.

The main tool in our approach to the differential treatment of automorphic functions will be the use of Fuchs theory, together with a rational ordinary differential operator of order three, obtained by a suitable modification of an operator introduced by H. A. Schwarz [12].

Definition 3.2. Let $f(z)$ denote a non-constant smooth function.

1. The schwarzian derivative of $f$ is defined as

$$
D s(f, z)=\frac{2 D(f, z) D^{3}(f, z)-3 D^{2}(f, z)^{2}}{D(f, z)^{2}} .
$$

2. The automorphic derivative of $f$ is defined as

$$
D a(f, z)=\frac{D s(f, z)}{D(f, z)^{2}} .
$$

A multivalued function defined on $\mathbb{P}^{1}$ is said to be $\mathbf{P G L}(2, \mathbb{C})$-multivalued if any pair of its branches is always projectively related. Examples of PGL $(2, \mathbb{C})$-multivalued functions occur by inversion of automorphic functions.

Proposition 3.3. Let $f(z), g(z)$ denote non-constant smooth functions whose composition $g \circ f$ is defined. The automorphic derivative satisfies the chain rule:

$$
D a(g \circ f, z)=D a(g, f(z))+\frac{D a(f, z)}{D(g, f(z))^{2}} .
$$

Proposition 3.4. Suppose that $f(z)=x$ is a smooth function whose inverse function is $\mathbf{P G L}(2, \mathbb{C})$-multivalued. Then the following assertions hold.

1. The schwarzian derivative $D s\left(f^{-1}, x\right)$ is univalued and

$$
D s\left(f^{-1}, x\right)=-D a(f, z), \quad f^{-1}(x)=z .
$$


2. The automorphic derivative $D a(f, z)$ of a $\Gamma$-automorphic function, $f(z)$, is again a $\Gamma$-automorphic function. That is to say, the following equality holds:

$$
\operatorname{Da}(f, \gamma(z))=\operatorname{Da}(f, z), \text { for all } \gamma \in \Gamma \text {. }
$$

As a consequence of the above proposition, we see that automorphic derivatives fit to automorphic functions and schwarzian derivatives fit to their inverses.

The following theorem, due to Poincaré, recurs throughout the papers in [10].

TheOrem 3.5 (Poincaré). Let $\Gamma$ be a fuchsian group of the first kind. Let $f(z)=x$ be a non-constant $\Gamma$-automorphic function and $z=f^{-1}(x)$ be its inverse. Then the functions

$$
\eta_{1}(x):=\frac{f^{-1}(x)}{D\left(f^{-1}, x\right)^{1 / 2}}, \quad \eta_{2}(x):=\frac{1}{D\left(f^{-1}, x\right)^{1 / 2}}
$$

yield a fundamental system of solutions of a linear differential equation

$$
D^{2}(\eta, x)+S(x, y) \eta=0 .
$$

Here $S(x, y)$ stands for a rational function. The functions $x, y$ are related by means of an algebraic equation

$$
F(X, Y)=0 .
$$

We see from the theorem that the multivalued function $f^{-1}$ can be obtained as a quotient of two fundamental solutions of a linear differential equation of the second order. In its turn, the automorphic function $f$ can be obtained as a solution of the non-linear equation of the third order

$$
D a(x, z)+S(x, y)=0 .
$$

Therefore, in order to calculate an automorphic function $f$ it suffices to know its automorphic derivative $-S(x, y)$. However, this can be a rather difficult task.

Let us restrict ourselves to the case of genus $g=0$. Then there exists a rational function $v$ in $\mathbb{C}(x, y)$ which generates this field over $\mathbb{C}$. Therefore, there exists a rational function $R(v)$ such that $S(x, y)=R(v)$.

Suppose that we are aware of a fundamental domain for the $\Gamma$-action on $\mathcal{H}$. If this is given by a $z$-polygon $\mathcal{P}$ whose sides are identified by pairs and whose internal angles equal $\alpha_{i} \pi$, then

$$
R(v)=\sum \frac{1-\alpha_{i}^{2}}{\left(v-a_{i}\right)^{2}}+\sum \frac{A_{i}}{v-a_{i}}
$$

as can be easily verified. The summation extends over all singular values $a_{i}$ of $v$, and $A_{i}$ are constants. If $\infty$ is an ordinary value of $v$, then

i) $\sum A_{i}=0$,

ii) $\sum a_{i} A_{i}+\sum\left(1-\alpha_{i}^{2}\right)=0$,

iii) $\sum a_{i}^{2} A_{i}+\sum a_{i}\left(1-\alpha_{i}^{2}\right)=0$.

If $\infty$ is a singular value of $x$ corresponding to a vertex of the polygon with angle equal to $\kappa \pi$, then

i) $\sum A_{i}=0$,

ii) $\sum a_{i} A_{i}+\sum\left(1-\alpha_{i}^{2}\right)-\left(1-\kappa^{2}\right)=0$. 
In general, these relations do not suffice to determine all the constants involved. The key point is that, in some cases, the symmetries of the function $v(z)$ we are looking for may allow a complete determination of $R(v)$.

4. Dedekind's valence function. Next we will examine Dedekind's valence function in accordance with the scheme of the section above.

Dedekind (op. cit.) starts with an operator

$$
[v, z]:=\frac{-4}{\sqrt{\frac{d v}{d z}}} \frac{d^{2}}{d v^{2}} \sqrt{\frac{d v}{d z}}
$$

which, in our notation, corresponds to

$$
[v, z]=-D a(v, z)=D s(z, v) .
$$

Next, he looks for a function $v: \mathcal{H} \rightarrow \mathbb{P}^{1}$ which, being invariant under the action of $\operatorname{PSL}(2, \mathbb{Z})$, satisfies

$$
v(i)=1, \quad v\left(e^{\frac{2 \pi i}{3}}\right)=0, \quad v(\infty)=\infty .
$$

From the observation of the fundamental hyperbolic triangle for the $\mathbf{S L}(2, \mathbb{Z})$-action on the upper-half plane, he deduces an equality of the form

$$
[v, z]=\frac{3}{4(v-1)^{2}}+\frac{8}{9 v^{2}}+\frac{A_{1}}{v-1}+\frac{A_{2}}{v} .
$$

The constants $A_{i}$ are determined by taking into account the behaviour at infinity (cf. section 3). We obtain

$$
[v, z]=\frac{36 v^{2}-41 v+32}{36 v^{2}(1-v)^{2}} .
$$

In order to recognize the valence function, Dedekind shows that the function

$$
\omega:=\text { const. } v^{-\frac{1}{3}}(1-v)^{-\frac{1}{4}}\left(\frac{d v}{d z}\right)^{\frac{1}{2}}
$$

satisfies the hypergeometric equation

$$
v(1-v) \frac{d^{2} \omega}{d v^{2}}+\left(\frac{2}{3}-\frac{7}{6} v\right) \frac{d \omega}{d v}-\frac{\omega}{144}=0 .
$$

Its solutions are linear combinations of the Gauss hypergeometric series

$$
F(1 / 12,1 / 12,2 / 3 ; v), \quad F(1 / 12,1 / 12,1 / 2 ; 1-v) .
$$

Moreover, since the function $v$ has to be invariant under the translation $z \mapsto z+1$, it may be developed as a power series in the variable $q=e^{2 \pi i z}$, already considered by Jacobi.

In fact, Dedekind goes much further in that paper. He justifies the existence of a polynomial equation, $F_{N}(X, Y)=0$, with integral coefficients, which is fulfilled by the pair of functions $(v(z), v(z / N))$.

For our purposes, we will consider a formal series $v(q)=\sum_{-1}^{\infty} C_{n} q^{n}$, with indeterminate coefficients, and compute them by integration of the differential equation of the third order

$$
D a(v, q)+\frac{36 v^{2}-41 v+32}{36 v^{2}(1-v)^{2}}=0 .
$$


Then, from a direct computation we will obtain

$$
v(q)=1728 j(q) .
$$

If we are interested in the differential equation satisfied by the $j$-function, it suffices to integrate the differential equation

$$
D a(j, q)+R(j)=0,
$$

where

$$
R(j)=\frac{j^{2}-1968 j+2654208}{j^{2}(j-1728)^{2}} .
$$

This is obtained through the initial conditions

$$
j(i)=1728, \quad j\left(e^{\frac{2 \pi i}{3}}\right)=0, \quad j(\infty)=\infty .
$$

5. Quaternion algebras, orders and fuchsian groups. In this section, we present some examples of arithmetic fuchsian groups obtained through quaternion orders. The details of their calculation can be found in [1].

Let $a, b$ be non-zero rational numbers. We consider the quaternion algebra

$$
H=\left(\frac{a, b}{\mathbb{Q}}\right)=\langle 1, i, j, k\rangle ; \quad i^{2}=a, \quad j^{2}=b, \quad i j=-j i=k,
$$

defined over the rational field $\mathbb{Q}$. We shall suppose that $H$ is indefinite, with $a>0$. Accordingly, $H$ may be embedded in the matrix algebra $\mathbf{M}(2, \mathbb{R})$, of the real $2 \times 2$ matrices. We shall fix the embedding $\Phi: H \hookrightarrow \mathbf{M}(2, \mathbb{R})$ defined as

$$
\Phi(x+y i+x j+t k)=\left[\begin{array}{cc}
x+y \sqrt{a} & z+t \sqrt{a} \\
b(z-t \sqrt{a}) & x-y \sqrt{a}
\end{array}\right] .
$$

Let $\mathbb{Q}_{p}$ denote the field of the $p$-adic numbers. A place $p$ of $\mathbb{Q}$ is said to ramify in $H$ if $H \otimes \mathbb{Q}_{p}$ is a division algebra. The discriminant $D_{H}$ of $H$ is defined as the product of all the places where the algebra $H$ ramifies:

$$
D_{H}=\prod_{v_{p} \text { ram }} p
$$

Since we have supposed that $H$ is indefinite, it is unramified at infinity and $D_{H}$ equals the product of an even number of different primes.

EXAmples 5.1. 1. For $D=1$, the algebra $H:=\left(\frac{1,-1}{\mathbb{Q}}\right)$ is isomorphic to the rational matrix algebra $M(2, \mathbb{Q})$. This is the only case in which $H$ has zero divisors and is not a division algebra.

2. The quaternion algebra $H_{A}(p):=\left(\frac{p,-1}{\mathbb{Q}}\right)$, where $p \equiv 3(\bmod 4)$ is a prime number, has discriminant $D=2 p$. The lowest values are $D=6$ and $D=14$.

3. The quaternion algebra $H_{B}(p, q):=\left(\frac{p, q}{\mathbb{Q}}\right)$, where $p, q$ are prime numbers, $q \equiv$ $1(\bmod 4)$ and the Legendre symbol $\left(\frac{p}{q}\right)$ is equal to -1 , has discriminant $D=p q$. The lowest values are $D=10$ and $D=15$.

By definition, an order of a quaternion algebra is a subring $\mathcal{O} \subseteq H$ which is of $\mathbb{Z}$-rank equal to 4 . The so-called Eichler orders are obtained as the intersection of two maximal 
orders. Their elements are integral over $\mathbb{Z}$. They are classified by their level, which is an integer $N$ prime to $D$. In our case, Eichler orders of level $N$ are determined up to conjugation in $H$. We shall denote by $\mathcal{O}(D, N)$ a representative in their conjugation class. The orders $\mathcal{O}(D, 1)$ are maximal.

EXAMPLES 5.2. The following examples correspond to Eichler orders of level $N$ square-free for the quaternion algebras considered above.

1. If $H=M(2, \mathbb{Q})$, take

$$
\mathcal{O}_{0}(1, N)=\left\{\left[\begin{array}{cc}
a & b \\
c N & d
\end{array}\right]: a, b, c, d \in \mathbb{Z}\right\}
$$

2. If $H=H_{A}(p)$, take

$$
\mathcal{O}_{A}(2 p, N)=\mathbb{Z}\left[1, i, N j, \frac{1+i+j+k}{2}\right], N \mid \frac{p-1}{2} .
$$

3. If $H=H_{B}(p, q)$, take

$$
\mathcal{O}_{B}(p q, N)=\mathbb{Z}\left[1, N i, \frac{1+j}{2}, \frac{i+k}{2}\right], N \mid \frac{q-1}{4}, \operatorname{gcd}(N, p)=1 .
$$

The subgroup of the multiplicative group of $\mathcal{O}_{0}(1, N)$ consisting of those matrices with determinant equal to one is the well-known congruence group of level $N, \Gamma_{0}(N)$. It is a fuchsian group of the first kind.

More generally, quaternion orders are a source for fuchsian groups. For an Eichler order $\mathcal{O}(D, N)$, the subgroup of the multiplicative group consisting of those quaternions of reduced norm equal to one, $\Gamma(D, N)$, is also a fuchsian group of the first kind. All these groups belong to a wider class: that of the arithmetic fuchsian groups.

EXAMPLES 5.3. 1. The group of units of reduced norm equal to one of the order $\mathcal{O}_{0}(1, N)$ is given by

$$
\Gamma(1, N)=\Gamma_{0}(N)=\left\{\gamma=\left[\begin{array}{cc}
a & b \\
N c & d
\end{array}\right]: a, b, c, d \in \mathbb{Z}, \operatorname{det} \gamma=1\right\} .
$$

2. The group of units of reduced norm equal to one of the order $\mathcal{O}_{A}(2 p, N)$ is given by

$$
\begin{aligned}
\Gamma(2 p, N)= & \left\{\gamma=\frac{1}{2}\left[\begin{array}{cc}
\alpha & \beta \\
-\beta^{\prime} & \alpha^{\prime}
\end{array}\right]: \alpha, \beta \in \mathbb{Z}[\sqrt{p}]\right. \\
& \left.\alpha \equiv \beta \equiv \alpha \sqrt{p}(\bmod 2), N \mid\left(\operatorname{tr}(\beta)-\frac{\beta-\beta^{\prime}}{\sqrt{p}}\right), \operatorname{det} \gamma=1\right\} .
\end{aligned}
$$

Here $\alpha^{\prime}$ denotes the galois conjugate of an element $\alpha$ in the field $\mathbb{Q}(\sqrt{p})$.

3. The group of units of norm equal to one of the order $\mathcal{O}_{B}(p q, N)$ is given by

$$
\begin{aligned}
\Gamma(p q, N)= & \left\{\gamma=\frac{1}{2}\left[\begin{array}{cc}
\alpha & \beta \\
q \beta^{\prime} & \alpha^{\prime}
\end{array}\right]: \alpha, \beta \in \mathbb{Z}[\sqrt{p}]\right. \\
& \left.\alpha \equiv \beta(\bmod 2), N \mid \frac{\alpha-\alpha^{\prime}-\beta+\beta^{\prime}}{2 \sqrt{p}}, \operatorname{det} \gamma=1\right\} .
\end{aligned}
$$


6. Shimura curves and their moduli interpretation. The seminal work for the study of curves attached to quaternionic fuchsian groups is due to G. Shimura. From 1959 onwards, he established their moduli interpretation, characterized their canonical models and investigated the main properties fulfilled by their $L$-functions.

Associated to the congruence subgroup $\Gamma_{0}(N)$, we have the modular curve $X_{0}(N)$. It is a moduli space for pairs $(E, G)$ consisting of an elliptic curve $E$ and a cyclic subgroup $G \subseteq E$ of order $N$.

We keep the embedding $\Phi: H \hookrightarrow \mathbf{M}(2, \mathbb{R})$, choose a maximal order $\mathcal{O}(D, 1)$, and fix a positive involution $\alpha \rightarrow \alpha^{*}$ of $H$.

The curves associated to the fuchsian groups $\Gamma(D, N)$ are called quaternionic Shimura curves. Shimura proved that they possess a canonical model, $X(D, N)$, defined over $\mathbb{Q}$. The values of the canonical mapping

$$
j_{D, N}: \mathcal{H} \rightarrow X(D, N)(\mathbb{C})
$$

correspond to classes $[(A, i, \mathcal{C}, G)]$ of polarized abelian surfaces endowed with quaternionic multiplication (QM) and level structure. More precisely:

i) $A / \mathbb{C}$ is an abelian variety of dimension 2 .

ii) We have embeddings of rings $i: \mathcal{O}(D, 1) \hookrightarrow \operatorname{End}(A), H \hookrightarrow \operatorname{End}^{0}(A)=\operatorname{End}(A) \otimes \mathbb{Q}$.

iii) $\mathcal{C}$ is a weak polarization on $A$ whose Rosatti involution is compatible with the positive involution on $H$.

iv) $G$ is a cyclic $\mathcal{O}(D, 1)$-module of order $N^{2}$ contained in the set $A[N]$ of the $N$-torsion points of $A$.

To define complex multiplication points (CM) for Shimura curves, let us consider a quadratic imaginary number field

$$
F=\mathbb{Q}(\sqrt{d}), \quad d<0,
$$

and an order $R(d, m)$ of conductor $m$ in its ring of integers.

A point $[(A, i, \mathcal{C}, G)]$ is said to be a CM-point by $R(d, m)$ if, and only if,

$$
\operatorname{End}(A, i, \mathcal{C}, G) \simeq R(d, m) .
$$

If this is the case, we have that

$$
\operatorname{End}^{0}(A, i, \mathcal{C}, G)=F,
$$

and there exists an embedding $\varphi: F \hookrightarrow H$ such that

$$
H \otimes F \simeq \mathbf{M}(2, F),
$$

i. e. the field $F$ splits the algebra $H$.

Canonical models are characterized by their CM-points. The important diophantine property is that their coordinates lie in class fields: if a point $z$ of $\mathcal{H}$ is a fixed point of $\varphi\left(F^{*}\right)$, then

$$
j_{D, N}(z)=[(A, i, \mathcal{C}, G)] \in X(D, N)\left(F^{a b}\right),
$$

$F^{a b}$ being the maximal abelian extension of $F$. 
Although many important issues remain unexplored, an increasing amount of literature today is devoted to the study of Shimura curves. We mention some of the main results obtained up to now.

Integral models for Shimura curves have been studied by Y. Ihara (1969, 1971); Y. Morita (1981); H. Carayol (1986) and K. Buzzard (1997).

Equations for Shimura curves are known in only a few cases. The first examples were computed by A. Kurihara $(1974,1994)$ and also by B. W. Jordan and R. Livné (1981).

Uniformization problems in the complex case have been treated by Y. Ihara (1974); J. F. Michon (1980); D. Krammer (1996); N. Elkies (1998); M. Alsina (1999) and S. Johansson (2001).

The $p$-adic uniformization of Shimura curves has been studied by I. V. Cerednik (1976) and V.G. Drinfeld (1976).

Results on hyperelliptic and bielliptic Shimura curves have been obtained by N. Ishii (1975); J. F. Michon (1981); A. P. Ogg (1983, 1985) and V. Rotger (2002).

Connections of Shimura curves with modular curves and their applications to Fermat's Last Theorem are due to K. A. Ribet (1980, 1990); F. Diamond-R. Taylor (1994) and F. Diamond (1997). In fact, although they do not appear explicitly in Wiles' famous paper on FLT, their role in the proof cannot be ignored.

Applications of Shimura curves to the theory of error-correcting-codes were stressed by M. A. Tsfasman-S. G. Vlăduţ-T. Zink (1982). But, it should be pointed out that, in order to use them effectively, a more explicit knowledge of their closed fibres would be necessary.

Diophantine problems on Shimura curves over several fields have been solved by B. W. Jordan-R. Livné (1984-87, 1999); A. P. Ogg (1985); S. Kamienny (1990); N. Elkies (1998) and T. Sasaki (2000).

Connections with $p$-adic $L$-functions and the Birch and Swinnerton-Dyer conjecture have been developed by M. Bertolini-H. Darmon (1998).

7. A fundamental polygon for $X(6,1)$. Let us fix a quaternion algebra $H=\left(\frac{a, b}{\mathbb{Q}}\right)$. The Eichler theory on arithmetic of quaternion algebras deals with embeddings

$$
\varphi: R(d, m) \hookrightarrow \mathcal{O}(D, N)
$$

of quadratic orders in quaternion orders. It provides a useful algebraic tool to study some of the most basic properties of the curves $X(D, N)$. Once the theory is translated in terms of binary quadratic forms, it allows many explicit calculations. Embeddings with $d>0$ yield hyperbolic transformations $\gamma \in \Gamma(D, N)$. For $d<0$, they allow the computation of CM-points. For $d=-1,-3$, they give rise to elliptic points.

Let $\varepsilon$ be a fundamental unit of $\mathbb{Q}(\sqrt{a})$. Put $\xi=\varepsilon$ if its norm is equal to 1 , and $\xi=\varepsilon^{2}$ if its norm equals -1 . Then the first power $h^{s}$ of $h=\left[\begin{array}{c}\xi \\ \xi^{\prime}\end{array}\right]$ such that $h^{s} \in \Gamma(D, N)$ generates the isotropy group $\Gamma(D, N)_{\infty}$. Moreover, for each $r \in \mathbb{R}^{+}$, the ring-shaped region

$$
S\left(r, \xi^{2 s} r\right)=\left\{z \in \mathcal{H}: r \leq|z| \leq \xi^{2 s} r\right\}
$$

is a fundamental domain for the group $\Gamma(D, N)_{\infty}$. 
The following theorem summarizes the main properties of a fundamental polygon for the Shimura curve $X(6,1)$.

Theorem 7.1 (Alsina [1], cf. Figure 1)).

1. The hyperbolic hexagon $\mathcal{P}(6,1)$ whose vertices are given by

$$
\begin{array}{ll}
P_{1}=\frac{-\sqrt{3}+\iota}{2}, & P_{2}=\frac{-1+\iota}{1+\sqrt{3}}, \\
P_{3}=(2-\sqrt{3}) \iota, & P_{4}=\frac{1+\iota}{1+\sqrt{3}}, \\
P_{5}=\frac{\sqrt{3}+\iota}{2}, & P_{6}=\iota,
\end{array}
$$

where $\iota^{2}=-1$, is a fundamental polygon for the action of $\Gamma(6,1)$ on $\mathcal{H}$.

2. All the vertices in 1 . are elliptic. The corresponding elliptic transformations fixing them are equal to

$$
\begin{array}{ll}
\gamma_{P_{1}}=\left[\begin{array}{cc}
\sqrt{3} & 2 \\
-2 & -\sqrt{3}
\end{array}\right], & \gamma_{P_{2}}=\frac{1}{2}\left[\begin{array}{cc}
1+\sqrt{3} & 3-\sqrt{3} \\
-3-\sqrt{3} & 1-\sqrt{3}
\end{array}\right], \\
\gamma_{P_{3}}=\left[\begin{array}{cc}
0 & -2+\sqrt{3} \\
2+\sqrt{3} & 0
\end{array}\right], & \gamma_{P_{4}}=\frac{1}{2}\left[\begin{array}{cc}
1+\sqrt{3} & -3+\sqrt{3} \\
3+\sqrt{3} & 1-\sqrt{3}
\end{array}\right], \\
\gamma_{P_{5}}=\left[\begin{array}{cc}
\sqrt{3} & -2 \\
2 & -\sqrt{3}
\end{array}\right], & \gamma_{P_{6}}=\left[\begin{array}{cc}
0 & 1 \\
-1 & 0
\end{array}\right] .
\end{array}
$$

3. The hyperbolic volume of $\mathcal{P}(6,1)$ is given by

$$
\int_{\Gamma(6,1) \backslash \mathcal{H}} \frac{d x \wedge d y}{y^{2}}=\frac{2 \pi}{3} .
$$

The curve $X(6,1)$ is of genus $g(6,1)=0$.

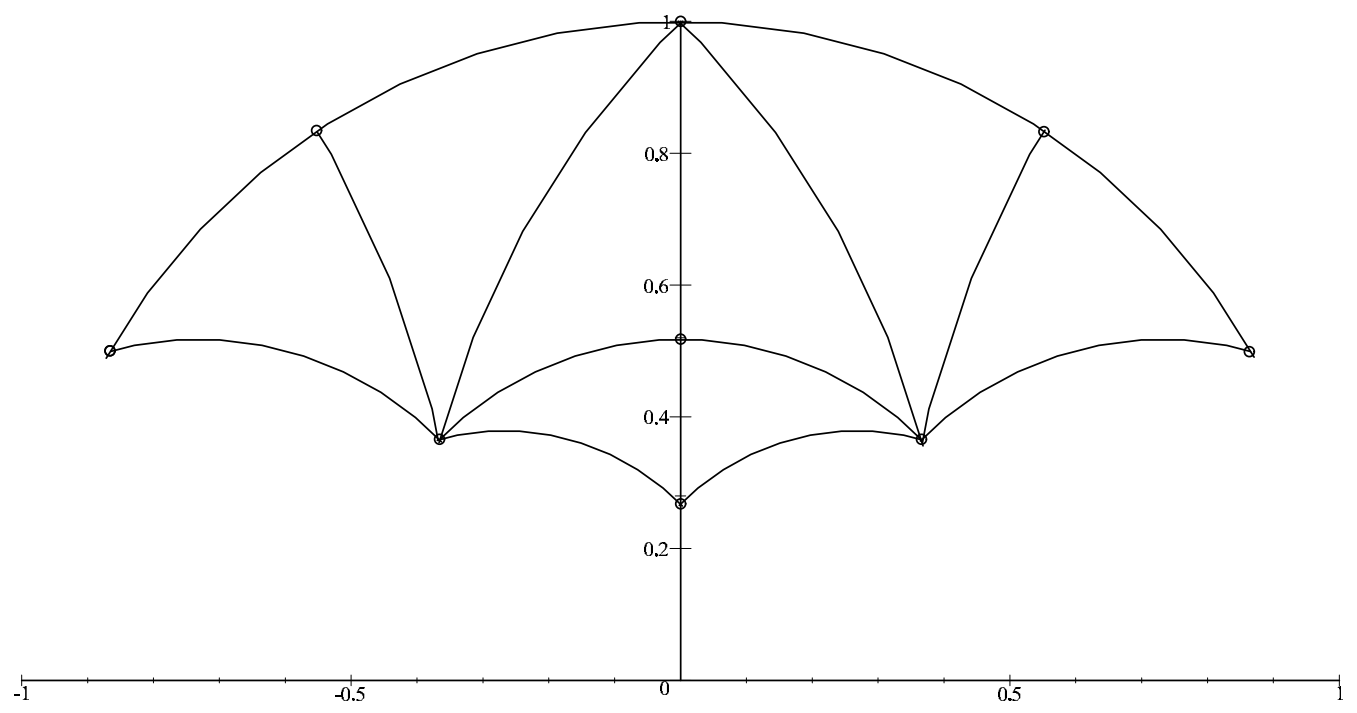

Fig. 1 
4. The isotropy group $\Gamma(6,1)_{\infty}$ is generated by

$$
h=\left[\begin{array}{cc}
2+\sqrt{3} & 0 \\
0 & 2-\sqrt{3}
\end{array}\right] .
$$

5. The fundamental polygon $\mathcal{P}(6,1)$ is included in $S\left(r_{1}, r_{3}\right)$ and $S\left(r_{2}, r_{4}\right)$, both fundamental domains for $\Gamma(6,1)_{\infty}$, where $r_{1}=7-4 \sqrt{3}, r_{2}=2-\sqrt{3}$, and $r_{3}=1, r_{4}=$ $2+\sqrt{3}$.

6. The sides of $\mathcal{P}(6,1)$ are identified in pairs: $\left(P_{2} P_{3}, P_{2} P_{1}\right)$ by $\gamma_{P_{2}},\left(P_{3} P_{4}, P_{5} P_{4}\right)$ by $\gamma_{P_{4}}$, and $\left(P_{5} P_{6}, P_{1} P_{6}\right)$ by $\gamma_{P_{6}}$.

7. The polygon $\mathcal{P}(6,1)$ contains the following elliptic cycles: $\left\{P_{1}, P_{3}, P_{5}\right\}$, of order $e=2 ;\left\{P_{2}\right\},\left\{P_{4}\right\}$, of order $e=3 ;\left\{P_{6}\right\}$, of order $e=2$.

8. The group $\bar{\Gamma}(6,1)=\Gamma(6,1) /\langle-\mathrm{I}\rangle$ can be described by generators and relations in accordance with

$$
\left\langle\gamma_{P_{2}}, \gamma_{P_{4}}, \gamma_{P_{6}}: \gamma_{P_{2}}^{3}=\gamma_{P_{4}}^{3}=\gamma_{P_{6}}^{2}=\left(\gamma_{P_{2}}^{-1} \gamma_{P_{6}} \gamma_{P_{4}}\right)^{2}=\mathrm{I}\right\rangle
$$

9. The CM-points of parameters $d=6$ and $m=1$ (called special) are

$$
\begin{gathered}
P_{0}=\frac{(\sqrt{6}-\sqrt{2}) \iota}{2}, \\
P_{7}=\frac{-\sqrt{3}+\sqrt{6} \iota}{3} \sim P_{8}=\frac{\sqrt{3}+\sqrt{6} \iota}{3} .
\end{gathered}
$$

10. The polygon $\mathcal{P}(6,1)$ is invariant under the involution

$$
w_{6}:=\frac{1}{\sqrt{6}}\left[\begin{array}{cc}
0 & -3+\sqrt{3} \\
3+\sqrt{3} & 0
\end{array}\right] .
$$

8. Uniformization of $X(6,1)$. In this section, we indicate a possible way to calculate a uniformizing function $j_{6,1}$ for the curve $X(6,1)$. The function $j_{6,1}$ has to be understood as an analogue of the modular function $j=j_{1,1}$.

The normalizer group $\bar{\Gamma}^{+}(6,1)$ of $\bar{\Gamma}(6,1)$ in $\operatorname{PSL}(2, \mathbb{R})$ is obtained by adding to the group $\bar{\Gamma}(6,1)$ three involutions $w_{2}, w_{3}, w_{6}$. They are defined through quaternions of norm equal to 2,3 and 6 , respectively.

A closer examination of the fundamental polygon $\mathcal{P}(6,1)$ reveals that the $\bar{\Gamma}^{+}(6,1)$ action on $\mathcal{H}$ originates a splitting of $\mathcal{P}(6,1)$ in eight hyperbolic triangles. By using them appropriately, it is possible to obtain fundamental polygons for the three quotient curves

$$
\begin{gathered}
X^{\prime}(6,1):=X(6,1) /\left\langle w_{6}\right\rangle, \quad X^{\prime \prime}(6,1):=X(6,1) /\left\langle w_{2}\right\rangle, \\
X^{\prime \prime \prime}(6,1):=X(6,1) /\left\langle w_{3}\right\rangle,
\end{gathered}
$$

as well as for the quotient curve

$$
X^{+}(6,1):=X(6,1) /\left\langle w_{2}, w_{3}\right\rangle .
$$

The five fundamental polygons obtained in total give rise in turn to five differential equations of fuchsian type. Their constants have been computed to fit together.

By integrating the attached five differential equations of the third order, five automorphic functions have been obtained, which also depend on certain constants. A careful observation of these functions in the neighbourhood of the special CM-points, and of 
the vertices of $P(6,1)$, makes it possible to write the necessary initial conditions to be fulfilled.

At this point, it only remains to choose the right variable to develop the solutions above in power series. When this is done, the functions appear with rational coefficients. In this way we obtain

$$
\begin{aligned}
j_{(6,1)}(q)= & 6 q-\frac{60}{3 !} q^{3}-\frac{98352}{5 !} q^{5}+\frac{31583520}{7 !} q^{7}+\frac{32129374464}{9 !} q^{9} \\
& -\frac{50955333603840}{11 !} q^{11}-\frac{39712797584898048}{13 !} q^{13} \\
& +\frac{276361426443834593280}{15 !} q^{15}-\frac{36203401544839069630464}{17 !} q^{17} \\
& +\mathrm{O}\left(q^{19}\right)
\end{aligned}
$$

where $q=i \pi_{(6,1)} \frac{z-P_{0}}{z+P_{0}}$.

\section{References}

[1] M. Alsina, Aritmètica d'ordres quaterniònics i uniformització hiperbòlica de corbes de Shimura. Tesis. Universitat de Barcelona, ISBN: 84-475-2491-4, 1999.

[2] D. V. Chudnovsky and G. V. Chudnovsky, Trascendental methods and theta functions, in: Proc. Symp. Pure Math. 49, Amer. Math. Soc., 1989, 167-232.

[3] R. Dedekind, Schreiben an Herrn Borchardt über die Theorie der elliptischen ModulFunctionen, J. Reine Angew. Math. 83 (1877), 265-292.

[4] N. D. Elkies, Shimura curves computations, in: Lecture Notes in Computer Sciences 1423, Springer, 1998, 1-49.

[5] A. R. Forsyth, Theory of Differential Equations, vol. IV, Cambridge Univ. Press, 1902.

[6] L. Fuchs, Zur Theorie der linearen Differentialgleichungen mit veränderlichen Coefficienten, J. Reine Angew. Math. 66 (1866), 121-160.

[7] Y. Ihara, Schwarzian equations, J. Fac. Sci. Univ. Tokyo 21 (1974), 97-118.

[8] D. Krammer, An example of an arithmetic Fuchsian group, J. Reine Angew. Math. 473 (1996), 69-85.

[9] J. Lehner, Discontinuous Groups and Automorphic Functions, Math. Surveys Monographs, 8, AMS, 1964.

[10] H. Poincaré, Euvres de Henri Poincaré, t. II, Gauthier-Villars, 1916.

[11] G. Shimura, Introduction to the Arithmetic Theory of Automorphic Functions, Iwanami Shoten and Princeton Univ. Press, 1971.

[12] H. A. Schwarz, Ueber diejenigen Fälle, in welchen die Gaussische hypergeometrische Reihe eine algebraische Function ihres vierten Elementes darstellt, J. Reine Angew. Math. 75 (1873), 292-335.

[13] K. Takeuchi, Arithmetic triangle groups, J. Math. Soc. Japan 29 (1977), 91-106.

[14] M. Yoshida, Fuchsian Differential Equations, Max-Planck-Institut für Mathematik, Vieweg \& Sohn, 1987. 